Accepted by APJ

\title{
Infall and outflow detections in a massive core JCMT 18354-0649S
}

\author{
Tie Liu ${ }^{1}$, Yuefang $\mathrm{Wu}^{1}$, Qizhou Zhang ${ }^{2}$, Zhiyuan $\mathrm{Ren}^{1}$, Xin Guan ${ }^{1,3}$ and Ming Zhu ${ }^{4}$
}

\begin{abstract}
We present a high-resolution study of a massive dense core JCMT 183540649S with the Submillimeter Array. The core is mapped with continuum emission at $1.3 \mathrm{~mm}$, and molecular lines including $\mathrm{CH}_{3} \mathrm{OH}\left(5_{23}-4_{13}\right)$ and $\mathrm{HCN}(3-2)$. The dust core detected in the compact configuration has a mass of $47 M_{\odot}$ and a diameter of $2^{\prime \prime}(0.06 \mathrm{pc})$, which is further resolved into three condensations with a total mass of $42 M_{\odot}$ under higher spatial resolution. The HCN (3-2) line exhibits asymmetric profile consistent with infall signature. The infall rate is estimated to be $2.0 \times 10^{-3} M_{\odot} \cdot \mathrm{yr}^{-1}$. The high velocity $\mathrm{HCN}(3-2)$ line wings present an outflow with three lobes. Their total mass is $12 M_{\odot}$ and total momentum is $121 M_{\odot} \cdot \mathrm{km} \mathrm{s}^{-1}$, respectively. Analysis shows that the N-bearing molecules especially $\mathrm{HCN}$ can trace both inflow and outflow.
\end{abstract}

Subject headings: Massive core:pre-main sequence-ISM: molecular-ISM: kinematics and dynamics-ISM: jets and outflows-stars: formation

\section{Introduction}

Studies of high-mass star formation have received much attention during recent years. One of the main questions is whether massive stars form through an accretion-disk-outflow process, similar to low-mass counterparts (Shu, Adams, \& Lizano 1987), or via collisioncoalescence (Wolfire \& Cassinelli 1987; Bonnell, Bate, \& Zinnecker. 1998). Studying the

\footnotetext{
${ }^{1}$ Department of Astronomy, Peking University, 100871, Beijing China; liutiepku@gmail.com, ywu@pku.edu.cn

${ }^{2}$ Harvard-Smithsonian Center for Astronomy, 60 Garden St., Cambridge, MA 02138, USA

${ }^{3}$ Current affiliation: I. Physikal. Institut, Universität zu Köln, Zülpicher St.77,D-50937 Köln, Germany

${ }^{4}$ National Astronomical Observatories, Chinese Academy of Sciences, Beijing, 100012
} 
characteristics of massive cores at the early stages is critical for understanding their formation process. High-Mass Protostellar Objects (HMPOs) are precursors of UC HII regions, and represent an essential phase in high-mass star formation (Churchwell 2002). HMPOs often have strong dust emission and high bolometric luminosity. But their radio emission is weak or non detectable at a level of approximately 1 mJy (Molinari et al. 1996, 2000; Sridharan et al. 2002; Beuther, Schilke, \& Menten. 2002; Wu et al. 2006).. Their natal clouds have not been affected significantly by the star forming process. Thus, they present the information about the early kinematic processes of high mass star formation.

The dense core JCMT 18354-0649S was first detected in an ammonia survey of highmass star forming regions with Max-Planck-Institut für Radioastronomie (MPIfR) $100 \mathrm{~m}$ telescope at Effelsberg (Wu et al. 2006), and was later confirmed by the observation with the Submillimeter Common-User Bolometric Array (SCUBA) of James Clerk Maxwell telescope (JCMT) (Wu et al. 2005). Another SCUBA core which harbors a UC H II region G35.4NW is located about $1^{\prime}$ north of JCMT 18354-0649S. The kinetic distance of the two SCUBA cores is 5.7 or $9.6 \mathrm{kpc}$ (Wu et al. 2005), and $5.7 \mathrm{kpc}$ was adopted in this paper. Core JCMT 18354-0649S has no counterpart in radio continuum. Multiple lines towards this core including $\mathrm{HCN}(3-2), \mathrm{H}^{13} \mathrm{CO}^{+}(3-2)$, and $\mathrm{C}^{17} \mathrm{O}(2-1)$ reveal typical "blue profile" (Wu et al. 2005), indicating that the core is undergoing gravitational collapse (Keto, Ho \& Haschick. 1988; Zhou et al. 1993; Zhang, Ho, \& Ohashi. 1998; Wu \& Evans. 2003; Wu et al. 2005; Fuller, Williams, \& Sridharan. 2005; Wyrowski 2006; Wu et al. 2007; Birkmann et al. 2007; Klaassen \& Wilson 2007; Sun \& Gao 2008; Velusamy et al. 2008). The core is also associated with a near-infrared point source, corresponding to a star of 6-11 $M_{\odot}$ (Zhu et al.2010, submitted). Carolan et al. (2009) observed sixteen different molecular line transitions including $\mathrm{CO}, \mathrm{HCN}, \mathrm{HCO}^{+}$and their isotopes in this region, and modeled the source with a chemically depleted rotating envelope collapsing onto a central protostellar source which has evolved sufficiently to generate a molecular outflow. All the evidence suggests that JCMT 18354-0649S is forming high mass protostellar object(s)(HMPO). However, single-dish observations with a resolution of $15^{\prime \prime}-40^{\prime \prime}$ can not reveal detailed kinematics in the core at a distance as large as $5.7 \mathrm{kpc}$. In this paper we report the results of a high resolution study with the Submillimeter Array (SMA 1 ) in order to probe the details of the core and its kinematic features. The observation and initial results are presented in sections 2 and 3. Properties of infall and outflow motions are discussed further in section 4 , and a brief summary is given in section 5 .

\footnotetext{
${ }^{1}$ Submillimeter Array is a joint project between the Smithsonian Astrophysical Observatory and the Academia Sinica Institute of Astronomy and Astrophysics and is funded by the Smithsonian Institution and the Academia Sinica.
} 


\section{Observations}

The observations of JCMT 18354-0649S was carried out with the SMA in July 2005 with seven antennas in its compact configuration and in September 2005 with six antennas in its extended configuration. The $345 \mathrm{GHz}$ receivers were tuned to $265 \mathrm{GHz}$ for the lower sideband (LSB) and $275 \mathrm{GHz}$ for the upper sideband (USB). The frequency spacing across the spectral band is $0.8125 \mathrm{MHz}$ or $\sim 1 \mathrm{~km} \mathrm{~s}^{-1}$ for both configurations. The phase reference center of both observations was R.A.(J2000) $=18^{\mathrm{h}} 38^{\mathrm{m}} 08 \cdot 10^{\mathrm{s}}$ and DEC.(J2000) $=-6^{\circ} 46^{\prime} 52 \cdot 17^{\prime \prime}$.

In the observations with the compact configuration, Jupiter, Uranus and QSO 3c454.3 were observed for antenna-based bandpass correction. An amplitude offset was found on some baselines and the baseline-based errors in bandpass were further corrected using the point source QSO 3c454.3. QSOs 1741-038 and 1908-201 were employed for antenna-based gain correction. Uranus was observed for flux-density calibration. The synthesized beam size is $3.76^{\prime \prime} \times 2.72^{\prime \prime}\left(\mathrm{PA}=-54^{\circ}\right)$.

For the extended configuration, QSO 3c454.3 was used as bandpass calibrator, QSOs 1741-038 and 1908-201 as gain calibrators, and Uranus as a flux calibrator, respectively. The synthesized beam size is about $1^{\prime \prime}$.

Miriad 2 was employed for calibration and imaging. The $1.3 \mathrm{~mm}$ continuum data were acquired by averaging all the line-free channels over both the $2 \mathrm{GHz}$ of upper and lower spectral bands. MIRIAD task "selfcal" was employed to perform self-calibration on the continuum data. Since the dust emission is weak, self-calibration with phase only was performed. The gain solutions from the self-calibration were applied to the line data.

The continuum data combined from both configurations yield a synthesized beam of $1.63^{\prime \prime} \times 1.28^{\prime \prime}\left(\mathrm{PA}=-81.4^{\circ}\right)$, and $1 \sigma \mathrm{rms}$ of $2.5 \mathrm{mJy}$ in the naturally weighted maps. HCN (3-2) and $\mathrm{CH}_{3} \mathrm{OH}\left(5_{23}-4_{13}\right)$ were detected in the compact configuration.

The shortest baseline in compact configuration observations is $16.5 \mathrm{~m}$, corresponding to a spatial scale of $20^{\prime \prime}$. Spatial structures more extended than this limit, such as HCN maps close to the cloud velocity, would be filtered out. HCN (3-2) data in JCMT archive (Wu et al. 2005; Carolan et al. 2009) were used to recover the missing flux. The JCMT archive data were reduced using the KAPPA and GAIA packages in the STARLINK suite. The JCMT beam size for HCN (3-2) was 18.3", and the main beam efficiency was 0.69. The combination of the SMA compact and JCMT HCN (3-2) data was done using the task "immerge" in MIRIAD.

\footnotetext{
${ }^{2}$ http://carma.astro.umd.edu/miriad
} 


\section{Results}

\subsection{Dust core}

The $1.3 \mathrm{~mm}$ continuum images are shown in Fig.1. The left panel is obtained in the compact array and the right panel from the combined data of the compact and extended configurations. An elongated core is revealed with the $1.3 \mathrm{~mm}$ continuum emission observed with the compact array, and is further resolved into three condensations by the continuum emission using the combined data from both configurations. The three condensations are named as MM1, MM2 and MM3. The peak position of MM1 is R.A.(J2000) $=18^{\mathrm{h}} 38^{\mathrm{m}} 08 \cdot 1^{\mathrm{s}}$, DEC. $(J 2000)=-6^{\circ} 46^{\prime} 52.98^{\prime \prime}$. MM2 peaks at R.A.(J2000) $=18^{\mathrm{h}} 38^{\mathrm{m}} 07.9^{\mathrm{s}}$, DEC.(J2000) $=-6^{\circ} 46^{\prime} 51.36^{\prime \prime}$, and MM3 peaks at R.A.(J2000) $=18^{\mathrm{h}} 38^{\mathrm{m}} 08.05^{\mathrm{s}}$, DEC.(J2000) $=-6^{\circ} 46^{\prime} 51.36^{\prime \prime}$.

From the fit of an elliptical Gaussian, the core revealed by the compact array is found to be elongated from south-east to north-west. It has an average FWHM diameter of $0.06 \mathrm{pc}$ $\left(\sim 2^{\prime \prime}\right)$ at a distance of $5.7 \mathrm{kpc}$, smaller than the beam size of the compact configuration. The total integrated flux is $0.47 \mathrm{Jy}$. The total dust and gas mass can be obtained with the formula $M=S_{\nu} D^{2} / \kappa_{\nu} B_{\nu}\left(T_{d}\right)$, where $S_{\nu}$ is the flux at $1.3 \mathrm{~mm}, \mathrm{D}$ is the distance, and $B_{\nu}\left(T_{d}\right)$ is the Planck function. We adopt a dust opacity $k_{1330}=1.4 \times 10^{-2} \mathrm{~cm}^{2} \mathrm{~g}^{-1}$ at $1.3 \mathrm{~mm}$ calculated from Ossenkopf \& Henning (1994) with a dust opacity index $\beta=2$. Here the ratio of gas to dust is taken as 100. Molecular line $\mathrm{CH}_{3} \mathrm{OH}\left(5_{23}-4_{13}\right)$ is detected, and its emission peak coincides with the dust core very well (see Sec.3.2). The upper energy level of the $\mathrm{CH}_{3} \mathrm{OH}$ $\left(5_{23}-4_{13}\right)$ line is $57 \mathrm{~K}$ above the ground, indicating a relatively warm conditions. Assuming $T_{d}=57 \mathrm{~K}$, a total dust and gas mass of $47 M_{\odot}$ is derived. A beam-average gas/dust density amounts to $2.0 \times 10^{6} \mathrm{~cm}^{-3}$, which is larger than $1.1 \times 10^{6} \mathrm{~cm}^{-3}$ obtained from single-dish telescope (Wu et al. 2005).

The three condensations (MM1, MM2 and MM3) have total integrated flux of 0.42 Jy, leading to a total mass of $42 M_{\odot}$ (assuming $\mathrm{T}_{d}=57 \mathrm{~K}$ as above). MM1 has a diameter of $0.02 \mathrm{pc}$, and a mass of $30 M_{\odot}$. MM1 is centrally concentrated and compact, while MM2 and MM3 are much more diffuse and extended.

With UKIRT (United Kingdom Infrared Telescope) Zhu et al. (2010, in preparation) detected three near-infrared sources IRS1a, IRS1b, IRS1c in H, K and L bands. Their positions are marked with crosses in Fig.1. IRS1a $\left(18^{\mathrm{h}} 38^{\mathrm{m}} 08.135^{\mathrm{s}},-6^{\circ} 46^{\prime} 51.57^{\prime \prime}\right)$ lies about $1.6^{\prime \prime}$ north-east of MM1. IRS1b $\left(18^{\mathrm{h}} 38^{\mathrm{m}} 08.026^{\mathrm{s}},-6^{\circ} 46^{\prime} 56.24^{\prime \prime}\right)$ and IRS1c $\left(18^{\mathrm{h}} 38^{\mathrm{m}} 07.929^{\mathrm{s}}\right.$, $\left.-6^{\circ} 46^{\prime} 55.34^{\prime \prime}\right)$ are about $3^{\prime \prime}$ southwest from MM1 and are much fainter. 


\subsection{Gas core}

The molecular line $\mathrm{CH}_{3} \mathrm{OH}\left(5_{23}-4_{13}\right)$ is detected in the compact configuration. Fig.2 presents its spectrum at three positions and the integrated emission overlaid on the $1.3 \mathrm{~mm}$ continuum image. The central velocity of the $\mathrm{CH}_{3} \mathrm{OH}\left(5_{23}-4_{13}\right)$ spectra is $96.7 \mathrm{~km} \mathrm{~s}^{-1}$, which is taken as the systemic velocity of the core. The central velocity of $\mathrm{CH}_{3} \mathrm{OH}\left(5_{23}-\right.$ $4_{13}$ ) does not shift at different positions (see Fig.2), which should exclude rotation at the core. The P-V diagram of $\mathrm{CH}_{3} \mathrm{OH}\left(5_{23}-4_{13}\right)$ is shown in Fig.3, indicating a compact gas core without rotation. The emission center of $\mathrm{CH}_{3} \mathrm{OH}\left(5_{23}{ }^{-4_{13}}\right)$ (R.A.(J2000) $=18^{\mathrm{h}} 38^{\mathrm{m}} 08.092^{\mathrm{s}}$, DEC. $\left.(\mathrm{J} 2000)=-6^{\circ} 46^{\prime} 52.318^{\prime \prime}\right)$ coincides with MM1 very well. While there are no $\mathrm{CH}_{3} \mathrm{OH}$ components corresponding with MM2 and MM3. The deconvolved size of the gas core revealed by $\mathrm{CH}_{3} \mathrm{OH}\left(5_{23}-4_{13}\right)$ is $3.78^{\prime \prime} \times 2.76^{\prime \prime}\left(\mathrm{PA}=-29^{\circ}\right)$, comparable to the synthesized beam size of the compact array.

The HCN (3-2) (265.886GHz) spectra obtained from the SMA compact configuration and from the data combined from both compact and extended configurations are presented in the left panel of Fig.4. Both of the two spectra are averaged over a region of $5^{\prime \prime} \times 5^{\prime \prime}$, which show a redshifted absorption dip and broad wings. The line profiles observed with the SMA and JCMT, as well as a combination of the two are presented in the right panel of Fig.4. All the spectra in the right panel of Fig.4 are convolved with the JCMT beam (18.3") for comparing. One can see that the SMA compact array observations recover less than $10 \%$ of JCMT flux around the systematic velocity, but recover more than $30 \%$ flux at the wings. The combination of the SMA and JCMT data recovers more than $70 \%$ of the JCMT flux at all the velocity channels.

\subsection{Kinematic signatures of lines}

\subsubsection{Infall motion}

The left panel of Fig.4 shows the most prominent feature ("blue profile") of the HCN (3-2) line at the core. The absorption gap is more than $8 \mathrm{~km} \mathrm{~s}^{-1}$ wide, ranging from 93 to $101 \mathrm{~km} \mathrm{~s}^{-1}$. Fig.5 presents the channel maps of the HCN $(3-2)$ emission from $80 \mathrm{~km} \mathrm{~s}^{-1}$ to $109 \mathrm{~km} \mathrm{~s}^{-1}$ constructed from the combined data, which is convolved with the beam of the SMA compact configuration. The absorption is obvious in the velocity range $(95,99)$

$\mathrm{km} \mathrm{s}^{-1}$. The absorption dip is also clearly seen in the P-V diagrams (see Fig.6), which is much deeper than that revealed by the single-dish observation (Wu et al. 2005).

From the left panel of Fig.4, it is clearly to see that the centeral velocity of the absorption 
$\operatorname{dip}\left(98 \mathrm{~km} \mathrm{~s}^{-1}\right)$ is redshifted from the systematic velocity $\left(96.7 \mathrm{~km} \mathrm{~s}^{-1}\right)$ by $1.3 \mathrm{~km} \mathrm{~s}^{-1}$. Such a blue asymmetric line profile where the blue emission peak is at a higher intensity than the red one is a collapse signature of molecular cores (Zhou et al. 1993). The spectra constructed from JCMT data and the combined data (the right panel of Fig.4) also show significant "blue profile", confirming the existence of infall motions.

\subsubsection{Molecular outflow}

Besides the absorption dip, the HCN (3-2) line exhibits remarkable broad wings extending more than $40 \mathrm{~km} \mathrm{~s}^{-1}$. High-velocity gas also can be easily identified in P-V diagrams of the HCN (3-2) emission along the direction of P.A. $=15^{\circ}$ and P.A. $=90^{\circ}$ as shown in Fig.6. The HCN (3-2) emission obtained from SMA compact configuration is integrated from 80 to $87 \mathrm{~km} \mathrm{~s}^{-1}$ for the blue lobe and from 103 to $109 \mathrm{~km} \mathrm{~s}^{-1}$ for the red lobe, respectively. The contour map of the integrated flux are shown in Fig.7. As in the channel maps (Fig.5), we can see several clumps in each lobe in the integrated map. The integrated HCN (3-2) emission seems to comprise an S-shaped structure from north-east to south. Another jet-like structure extended more than $10^{\prime \prime}$ is also seen at the west of the continuum emission center. IRS1a seems to be the driving source of the outflow.

The southern redshifted lobe (S-lobe) comprises two clumps named "Clump1" and "Clump2". In the north-east blueshifted lobe (NE-lobe), two clumps are also found and named "Clump3" and "Clump4". These clumps are distributed along the direction of the outflow and likely to be outward gas knots. They are probably not physically related with other stellar sources except the driving source though Clump2 is close to IRS1b and IRS1c.

\section{Discussion}

\subsection{Infall motion}

Although the HCN emission is extended over a region larger than the compact configuration beam, the infall region is still difficult to confine due to the contamination of the outflow. Since the size of the gas core traced by $\mathrm{CH}_{3} \mathrm{OH}\left(5_{23}-4_{13}\right)$ is comparable to the compact configuration beam size, the beam size of the compact configuration was taken as the radius $\left(R_{i n}\right)$ of the infall region (Wu et al. 2009). The kinematic mass infall rate can be calculated using $\mathrm{dM} / \mathrm{dt}=4 \pi n \mu_{G} m_{H_{2}} R_{i n}^{2} V_{i n}$, where $V_{i n}, \mu_{G}=1.36, m_{H_{2}}$ and $\mathrm{n}=2.0 \times 10^{6} \mathrm{~cm}^{-3}$ are the infall velocity, the mean molecular weight, the $\mathrm{H}_{2}$ mass, and the beam-average gas/dust density, respectively. The infall velocity $V_{\text {in }}$ is $1.3 \mathrm{~km} \mathrm{~s}^{-1}$ by comparing the systemic velocity 
(96.7 $\left.\mathrm{km} \mathrm{s}^{-1}\right)$ and the velocity of the redshifted absorbing dip (98 $\left.\mathrm{km} \mathrm{s}^{-1}\right)$ in the HCN (3-2) spectrum (Welch et al. 1987), leading to a kinematic mass infall rate of $2.0 \times 10^{-3} M_{\odot} \cdot \mathrm{yr}^{-1}$. In core G10.6-0.4, the redshifted $\mathrm{NH}_{3}$ indicates large infall velocity $5.0 \pm 1.7 \mathrm{~km} \mathrm{~s}^{-1}$ at about $0.05 \mathrm{pc}$, and a mass infall rate as high as $5 \times 10^{-3} M_{\odot} \cdot \mathrm{yr}^{-1}$ (Keto, Ho \& Haschick. 1987). Also with $\mathrm{NH}_{3}$ inverse lines, Zhang \& Ho (1997) obtained a high infall velocity $\sim 3.5 \mathrm{~km} \mathrm{~s}^{-1}$ within a region smaller than $0.02 \mathrm{pc}$ towards core W51e2. Large infall velocities $\left(>1.5 \mathrm{~km} \mathrm{~s}^{-1}\right)$ and mass infall rates $\left(>1 \times 10^{-3} M_{\odot} \cdot \mathrm{yr}^{-1}\right)$ were also detected towards G10.47 and G34.26 with $\mathrm{HCO}^{+}$(4-3) line (Klaassen \& Wilson 2007). In core G19.61+0.23, an infall velocity of 2.5 $\mathrm{km} \mathrm{s}^{-1}$ and a mass infall rate as high as $6.1 \times 10^{-3} M_{\odot} \cdot \mathrm{yr}^{-1}$ were derived (Wu et al. 2009). It seems high mass infall rate is required by high-mass star formation. The results of the core JCMT 18354-0649S are comparable with those of the above sources. For comparison, the $V_{i n}$ from pure free-infall assumption is also derived with the formula $V_{i n}^{2}=2 G M / R_{\text {in }}$. The pure free-infall velocity inferred is $2.9 \mathrm{~km} \mathrm{~s}^{-1}$, larger than the infall velocity obtained from the spectrum.

Wu et al. (2005) obtained a small infall velocity $\left(\sim 0.3 \mathrm{~km} \mathrm{~s}^{-1}\right)$ at a radius of $4^{\prime \prime}$. The absorption dip of HCN (3-2) line seen by the SMA is much deeper and broader than

that observed by JCMT. However, the kinematic mass infall rate $\dot{M}_{i n}\left(2.0 \times 10^{-3} M_{\odot} \cdot \mathrm{yr}^{-1}\right)$ obtained here is well coincident with that obtained with JCMT (Wu et al. 2005), $3.4 \times$ $10^{-3} M_{\odot} \cdot \mathrm{yr}^{-1}$.

\subsection{Properties of HCN (3-2) outflow}

The column density of HCN at each velocity channel in each outflow lobe can be obtained through (Garden et al. 1991):

$$
N_{H C N}(v)=\frac{3 k}{8 \pi^{3} B \mu^{2}} \frac{\exp \left[h B J(J+1) / k T_{e x}\right]}{(J+1)} \frac{\left(T_{e x}+h B / 3 k\right)}{1-\exp \left(-h \nu / k T_{e x}\right)} \int \tau_{v} d v
$$

Where $v$ is the central velocity of the channel relative to the systemic velocity, the rotational constant $\mathrm{B}=44.315976 \mathrm{GHz}$ and permanent dipole moment $\mu=3$ debye for $\mathrm{HCN}$, the velocity channel width is smoothed to be $1 \mathrm{~km} \mathrm{~s}^{-1}$. Assuming HCN emission in the line wings to be optically thin and excitation temperature of $\mathrm{T}_{e x}=30 \mathrm{~K}$ (Wu et al. 2004), the optical depth $\tau_{v}$ can be derived with the equation:

$$
\tau_{v}=\frac{k T_{r}(v)}{h \nu}\left(\frac{1}{\exp \left(h \nu / k T_{e x}\right)-1}-\frac{1}{\exp \left(h \nu / k T_{b g}\right)-1}\right)^{-1}
$$

where $T_{r}(v)$ is the excess brightness temperature of $\mathrm{HCN}(3-2)$ emission at $v$. Adopting $X_{H C N}=[H C N] /\left[H_{2}\right]=1 \times 10^{-10}$ (Carolan et al. 2009), the mass of each lobe at $v$ can be 
calculated with:

$$
M(v)=X_{H C N}^{-1} \mu_{G} m_{H_{2}} D^{2} \int N_{H C N}(v) d \Omega
$$

where $\mathrm{D}, \Omega$ are the cloud distance and the solid angle. Thus the total mass of each lobe is given by $M=\sum M(v)$, the total momentum by $P=\sum M(v) v$, and the energy by $E=\frac{1}{2} \sum M(v) v^{2}$. The dynamical timescale $t_{d y n}$ is estimated as $R / V_{\max }$, where $\mathrm{R}$ is the outflow extent, and $V_{\max }$ is the maximum velocity of the outflow lobe. The mechanical luminosity L, and the mass-loss rate $\dot{M}$ are calculated as $\mathrm{L}=\mathrm{E} / \mathrm{t}, \dot{M}=P /\left(t V_{w}\right)$, where the wind velocity $V_{w}$ is assumed to be $500 \mathrm{~km} \mathrm{~s}^{-1}$ (Lamers et al. 1995). The derived parameters are listed in Table.1. The total mass, momentum, energy of the three lobes are $12 M_{\odot}$, $121 M_{\odot} \cdot \mathrm{km} \mathrm{s}^{-1}$ and $1.3 \times 10^{46} \mathrm{erg}$, respectively. The average dynamical timescale is about $1.6 \times 10^{4} \mathrm{yr}$, and the total mass-loss rate $1.6 \times 10^{-5} M_{\odot} \cdot \mathrm{yr}^{-1}$. The outflow is massive with parameters similar to that of IRAS $05274+3345 \mathrm{E}$ and the other outflows detected towards five massive star formation regions (Zhang et al. 2007b; Klaassen \& Wilson 2008).

The Position-Velocity diagram at the left panel of Fig. 6 shows that at low velocities, the NE-lobe and S-lobe both have compact morphology near the core center. At higher velocities the southern lobe becomes further away from the center. As shown in Fig.7, the outflow axis traced by Clump1 and Clump2 differs from that traced by Clump3 and Clump4. The different outflow orientations in the large and small scales may be attributed to the precession of the outflow axis (Su et al. 2007). From the right panel of the P-V diagram, a high-velocity component $\left(\mathrm{V}<85 \mathrm{~km} \mathrm{~s}^{-1}\right)$ with velocities decreasing with distance from the protostar, and a second component tracing the low-velocity material $\left(\mathrm{V}>85 \mathrm{~km} \mathrm{~s}^{-1}\right)$ extending about $15^{\prime \prime}$ along the axis of the W-lobe are clearly seen. Such convex spur PV structure was also revealed in a simulation of a pulsed jet driven outflow (Lee et al. 2001).

\subsection{HCN - tracer of both inflow and outflow motions}

HCN is among the most abundant molecular species with a high critical density larger than $10^{6} \mathrm{~cm}^{-3}$ (for HCN (1-0)) (Carolan et al. 2009), and is believed to trace dense molecular cores. HCN is detected in both low mass class 0 and I sources (Park, Kim, \& Ming. 1999; Yun et al. 1999), and high-mass hot cores (Boonman et al. 2001).

HCN is thought as a good tracer of inflow motions (Wu \& Evans. 2003). The infall asymmetry in the HCN spectra is found to be more prevalent, and more prominent than in any other previously used infall tracers such as CS $(2-1), \mathrm{DCO}^{+}(2-1)$, and $\mathrm{N}_{2} \mathrm{H}^{+}(1-0)$ during a survey toward 85 starless cores (Sohn et al. 2007). Among the small group of preand protostellar objects in L1251B, infall signature was also detected in the HCN emission 
(Lee et al. 2007). HCN also traces inflow motions very well in massive star-formation regions. Wu and Evans. (2003) found 12 sources showing "blue profile" in the HCN lines during a spectroscopic survey of 28 massive cores with water maser. Besides HCN, other nitrogen bearing molecules such as $\mathrm{N}_{2} \mathrm{H}^{+}$are also tracers of inflow motions (Tsamis et al. 2008; Schnee et al. 2007; Crapsi et al. 2005). Recently inverse P Cygni profile of CN line in hot cores was found (Zapata et al. 2008; Wu et al. 2009). These results suggest nitrogen bearing molecular species be good tracers of inflowing motions in star-formation regions.

Outflows traced by $\mathrm{HCN}$ are often detected not only in low-mass star-formation regions but also in massive star-formation regions (Bachiller, Gutiérrez, \& Pérez. 1997; Choi 2001; $\mathrm{Su}$ et al. 2007; Zhang et al. 2007a). HCN outflow of the core JCMT 18454-0649S is another good sample. Additionally, Zhu et al. (2010 in preparation) and Cyganowski (2008) found excess emission at $4.5 \mu \mathrm{m}$ at the position of source IRS1a, which is close to the center of the NE-lobe and S-lobe. Such excess emission at the $4.5 \mu \mathrm{m}$ band could be shock-excited. In IRAS 20126+4104, HCN emission is also found to be closely related to the shock-excited near-IR $\mathrm{H}_{2}$ knots and was identified to be associated with shock wings ( $\mathrm{Su}$ et al. 2007). The inner clumps (Clump1 in the S-lobe and Clump3 in the NE-lobe) of the core JCMT 183540649S should also be coincident with shocks. In fact, models have already demonstrated a dramatic increase of HCN molecules, during the intense interaction between outflow and ambient gas, or slow shock front (Mitchell 1984; Nejad, Williams, \& Charnley. 1990). In this process sulfur and nitrogen react with hydrocarbons to produce various compounds, wherein HCN abundance gets higher than the rest of the products (Nejad, Williams, \& Charnley. 1990). Thus HCN may trace the outflow even better than sulfur containing molecules.

\section{Summary}

Both dust continuum at $1.3 \mathrm{~mm}$ and $\mathrm{CH}_{3} \mathrm{OH}$ emission detected with SMA reveal a compact core in JCMT 18354-0649S. The core observed with the compact configuration has a mass of $47 M_{\odot}$ and an average density of $2.0 \times 10^{6} \mathrm{~cm}^{-3}$. With the combination of the compact and extended configurations, the core is resolved to three condensations with a total mass of $42 M_{\odot}$.

HCN (3-2) spectra exhibit an infall signature in this region. The red shifted absorption seen in the SMA observation is deeper and broader than that in the JCMT observation. The infall rate is $2.0 \times 10^{-3} M_{\odot} \cdot \mathrm{yr}^{-1}$. High velocity gas is detected in $\operatorname{HCN}(3-2)$ emission. The outflow has three lobes and their total mass is $12 M_{\odot}$ and momentum of $121 M_{\odot} \cdot \mathrm{km} \mathrm{s}^{-1}$. The average dynamical timescale and the total mass-loss rate are about $1.6 \times 10^{4} \mathrm{yr}$ and $1.6 \times 10^{-5} M_{\odot} \cdot \mathrm{yr}^{-1}$, respectively. All the findings indicate a high-mass protostar is forming 
via rapid accretion. Our results suggest that nitrogen bearing molecules especially HCN are good for probing both infall and outflows.

\section{Acknowledgment}

We are grateful to the SMA staff. We also thank Dr. Shengli Qin. for his help with

data reduction and discussion. This work was funded by Grants of NSFC No 10733030 and 10873019 .

\section{REFERENCES}

Arce, H. G., Shepherd, D., Gueth, F., Lee, C.-F., Bachiller, R., Rosen, A., Beuther, H., 2007, arXiv:astro-ph/0603071

Bachiller, R., Gutiérrez, M., \& Pérez. 1997, ApJ, 487, L93

Beuther, H., Schilke, P., \& Menten, K. M. 2002, ApJ, 566, 945

Birkmann, S. M., Krause, O., Hennemann, M., Henning, Th., Steinacker, J., Lemke, D. 2007, A\&A, 474, 883

Bonnell, I. A., Bate, M. R., \& Zinnecker, H. 1998, MNRAS, 298, 93

Boonman, A. M. S., Stark, R., Van der tak, F. F. S., Van dishoeck, E. F., Van der wal, P. B., Scháfer F., De lange, G., \& Laauwen, W. M. 2001, ApJ, 553, L63

Carolan, P. B., Khanzadyan, T., Redman, M. P., Thompson, M. A., Jones, P. A., Cunningham, M. R., Loughnane, R. M., Bains, I., Keto, E. 2009, MNRAS, 400, 78

Choi, Minho. 2001, ApJ, 553, 219

Churchwell, E. 2002, ARA\&A, 40, 27

Crapsi, A., Caselli, P., Walmsley, C., M., Myers, P., C., Tafalla, M., Lee, C., W., Bourke, T., L. 2005, ApJ, 619,379

Cyganowski, C. J. et al. 2008, AJ, 136, 2391

Fuller, G. A., Williams, S. J., \& Sridharan, T. K. 2005, A\&A, 442, 949 
Garden, R. P., Hayashi, M., Gatley, I., Hasegawa, T., \& Kaifu, N. 1991, ApJ, 374, 540

Keto, E. R., Ho, P. T. P., \& Haschick, A. D. 1987, apj, 318, 712

Keto, E. R., Ho, P. T. P., \& Haschick, A. D. 1988, ApJ. 324, 920

Klaassen, P. D. \& Wilson, C. D. 2007, ApJ, 663, 1092

Klaassen, P. D., \& Wilson, C. D. 2008, ApJ, 684, 1273

Lamers, H. J. G. L. M., Snow, T. P., Lindholm, D. M. 1995, ApJ, 455, 269

Lee, C.-F., Stone, J. M., Ostriker, E. C., \& Mundy, L. G. 2001, ApJ, 557, 429

Lee, J.-E., Di Francesco, J., Bourke, T. L., Evans, N. J. II \& Wu, J. 2007, ApJ, 671, 1748

Lester, D. F., Dinerstein, H. L., Werner, M. W., Harver, P. M., Evans, N. J. II, Brown, R. L. 1985, ApJ, 296, 565L

Mitchell, G. F. 1984, ApJ, 287, 665

Molinari, S., Brand, J., Cesaroni, R., Palla, F. 1996, A\&A, 308, 573

Molinari, S., Brand, J., Cesaroni, R., \& Palla, F. 2000, A\&A, 355, 617

Motoyama, K., \& Yoshida T. 2003, MNRAS, 344, 461

Myers, P. C., Mardones, D., Tafalla, M., Williams, J. P. \& Wilner, D. J. 1996, ApJ, 465, L133

Nejad, L. A. M., Williams, D. A., \& Charnley, S. B. 1990, MNRAS, 246, 183

Ossenkopf, V., Henning, Th. 1994, A\&A, 291, 943

Park, Y. S., Kim, J., \& Ming Y. C. 1999, ApJ, 520, 223

Schnee, S., Caselli, P., Goodman, A., Arce, H. G., Ballesteros-Paredes, J., Kuchibhotla, K. 2007, ApJ, 671, 1839

Shu, F. H. 1977, ApJ, 214, 488

Shu, F. H., Adams, F. C., \& Lizano, S. 1987, ARA\&A, 25, 23

Sohn, J. J., Lee, C. W., Park, Y.-S., Lee, H. M., Myers, P. C., \& Lee Y. 2007, ApJ, 664,928 
Sridharan, T. K., Beuther, H., Schilke, P., Menten, K. M., Wyrowski, F. 2002, ApJ, 566, 931

Su, Y.-N., Liu, S.-Y., Chen, H.-R., Zhang, Q., Cesaroni, R. 2007, ApJ, 671,571

Sun, Y., \& Gao, Y. 2008, MNRAS, 392, 170

Tsamis, Y. G., Rawlings, J. M. C., Yates, J. A., Viti, S. 2008, MNRAS, 388, 898

Velusamy, T., Peng, R., Li, D., Goldsmith, P. F., Langer, William D. 2008, ApJ, 688, L87

Welch, Wm. J., Dreher, J. W., Jackson, J. M., Terebey, S., Vogel, S. N. 1987, Science, 238, 1550

Wolfire, M. G. \& Cassinelli, J. P. 1987, ApJ, 319, 850

Wu, J., Evans N. J. II. 2003, ApJ, 592, L79

Wu, Y., Wei, Y., Zhao, M., Shi, Y., Yu, W., Qin, S., Huang, M. 2004, A\&A, 426, 503

Wu, Y., Zhu, M., Wei, Y., Xu, D., Zhang, Q., \& Fiege, J. D. 2005, ApJ, 628, L57

Wu, Y., Zhang, Q., Yu, W., Miller, M., Mao, R., Sun, K., \& Wang, Y. 2006, A\&A, 450, 607

Wu, Y., Henkel, C., Xue, R., Guan, X., Miller, M. 2007, ApJ, 669, L37

Wu, Y., Qin, S.-L., Guan, X., Xue, R., Ren, Z., Liu, T., Huang, M., Chen, S. 2009, ApJ, 697, L116

Wyrowski, F., Heyminck, S., Güsten, R., Menten, K. M. 2006, A\&A, 454, L95

Yun, J. L., Moreira, M. C., Afosso, J. M. \& Clemens, D. P. 1999, ApJ, 118, 990

Zapata, L. A., Palau, A., Ho, P. T. P., Schilke1, P., Garrod, R. T., Rodrguez, L. F., \& Menten, K. 2008, A\&A, 479, L25

Zhang, Q., \& Ho, P. T. P. 1997, ApJ, 488,241

Zhang, Q., Ho, P. T. P., \& Ohashi, N. 1998, ApJ, 494, 636

Zhang, Q., Sridharan, T. K., Hunter, T. R., Chen, Y.; Beuther, H., Wyrowski, F. 2007a, A\&A, 470, 269

Zhang, Q., Sridharan, T. K., Hunter, T. R., Chen, T., Beuther, H., \& Wyrowski, F. 2007b, ApJ, 658, 1152 
Zhou, S., Evans, N. J. II., Koempe, C., Walmsley, C. M. 1993, ApJ, 404, 232

This preprint was prepared with the AAS LATEX macros v5.2. 
Table 1: Outflow parameters of each lobe.

\begin{tabular}{cccccccc}
\hline \hline outflow & $\begin{array}{c}\mathrm{V}_{\max } \\
\mathrm{km} \mathrm{s}^{-1}\end{array}$ & $\begin{array}{c}t_{\text {dyn }} \\
\left(10^{4} \mathrm{yr}\right)\end{array}$ & $\begin{array}{c}\text { Mass } \\
\left(M_{\odot}\right)\end{array}$ & $\begin{array}{c}\text { momentum } \\
\left(M_{\odot} \mathrm{km} \mathrm{s}^{-1}\right)\end{array}$ & $\begin{array}{c}\text { Energy } \\
\left(10^{45} \mathrm{erg}\right)\end{array}$ & $\begin{array}{c}L \\
\left(L_{\odot}\right)\end{array}$ & $\begin{array}{c}\dot{M}_{\text {out }} \\
\left(10^{-6} M_{\odot} \mathrm{yr}^{-1}\right)\end{array}$ \\
\hline southern lobe & 11.7 & 1.4 & 3.3 & 29 & 3.0 & 1.8 & 4.0 \\
north-eastern lobe & 15.3 & 1.0 & 4.2 & 42 & 4.5 & 3.6 & 8.0 \\
western lobe & 15.3 & 2.3 & 4.6 & 50 & 5.5 & 2.1 & 4.0 \\
\hline
\end{tabular}




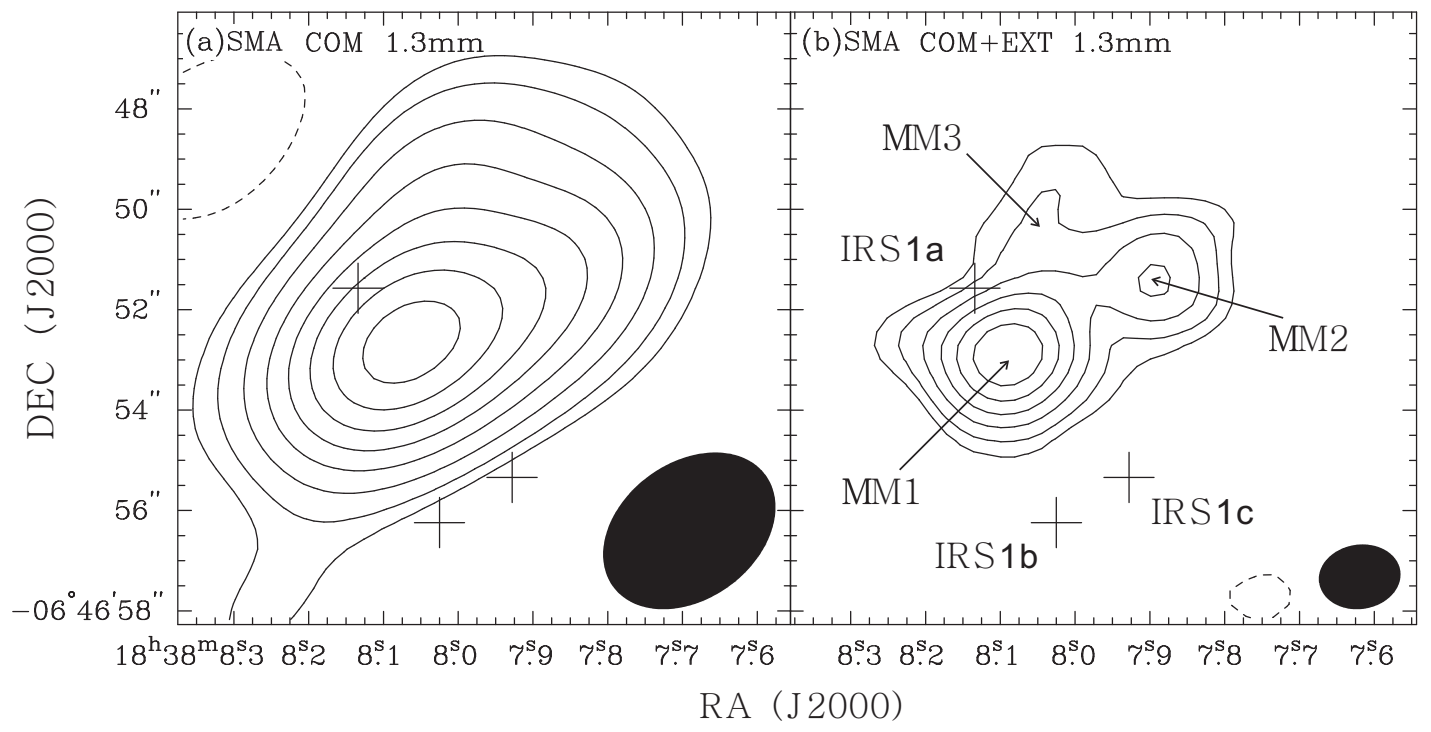

Fig. 1.- The $1.3 \mathrm{~mm}$ continuum emission towards JCMT 18354-0649S. The left one is obtained with the compact configuration. The rms level is $3 \mathrm{mJy}_{\mathrm{beam}}^{-1}(1 \sigma)$. The contours are at $-6,3,6,12,21,33,48,66,87 \sigma$. The right panel gives the contours of the continuum emission combined from both configurations. The rms level is $2.5 \mathrm{mJy} \mathrm{beam}^{-1}$ $(1 \sigma)$ and the contours are at $-6,3,6,12,21,33,48 \sigma$. The three near-infrared sources are marked with crosses. 


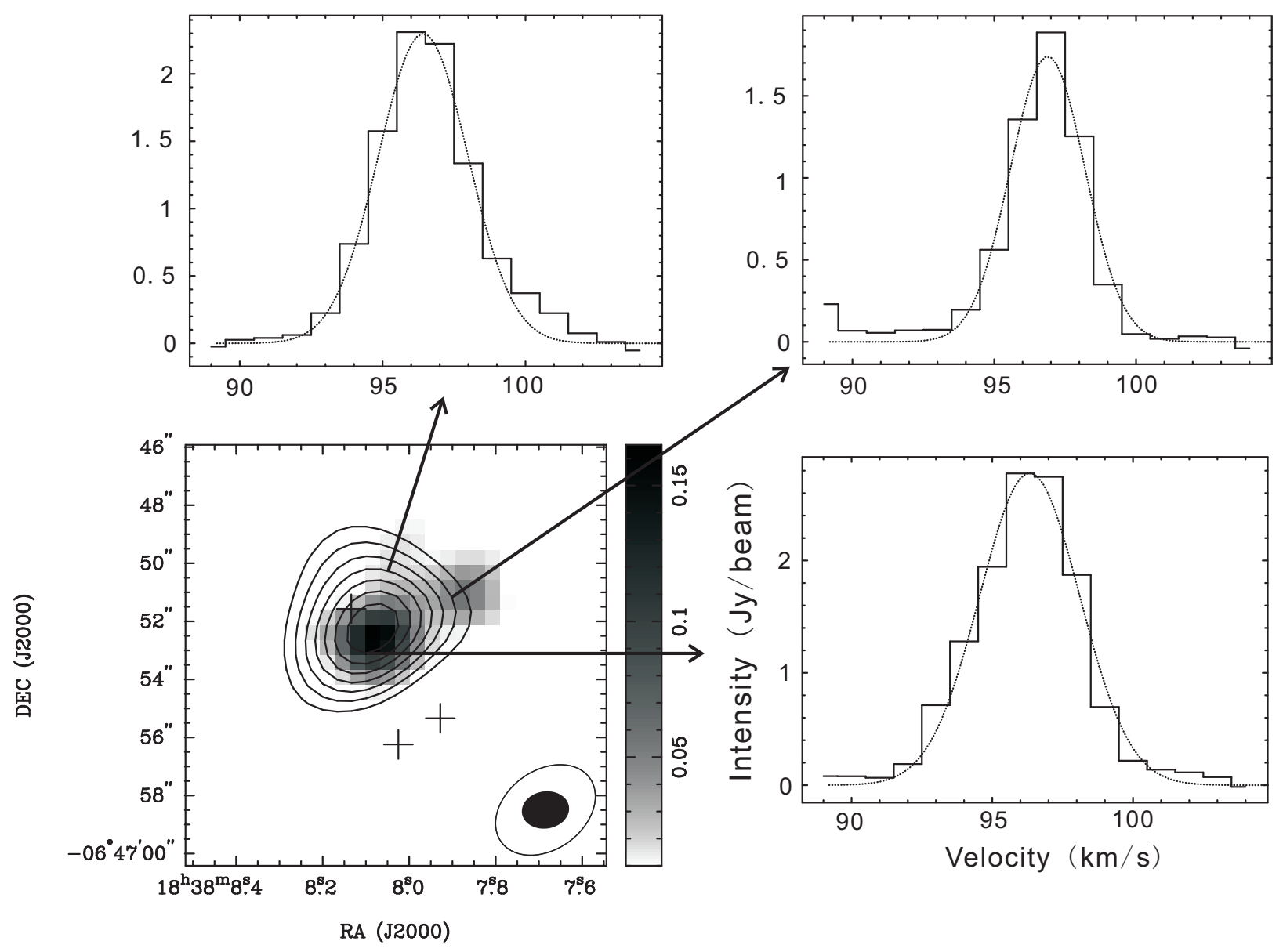

Fig. 2. - The lower-left panel is the contours of the $\mathrm{CH}_{3} \mathrm{OH}$ integrated intensity overlaid on the $1.3 \mathrm{~mm}$ continuum image (grey scale). The contours start from $30 \%$ in steps of $10 \%$ of the peak emission (15 Jy beam ${ }^{-1} \cdot \mathrm{km} \mathrm{s}^{-1}$ ). The three near-infrared sources are marked with crosses. The beam-averaged spectrum of $\mathrm{CH}_{3} \mathrm{OH}$ at three positions are presented in the other panels. The gaussian fit towards each spectrum is shown with solid lines. 


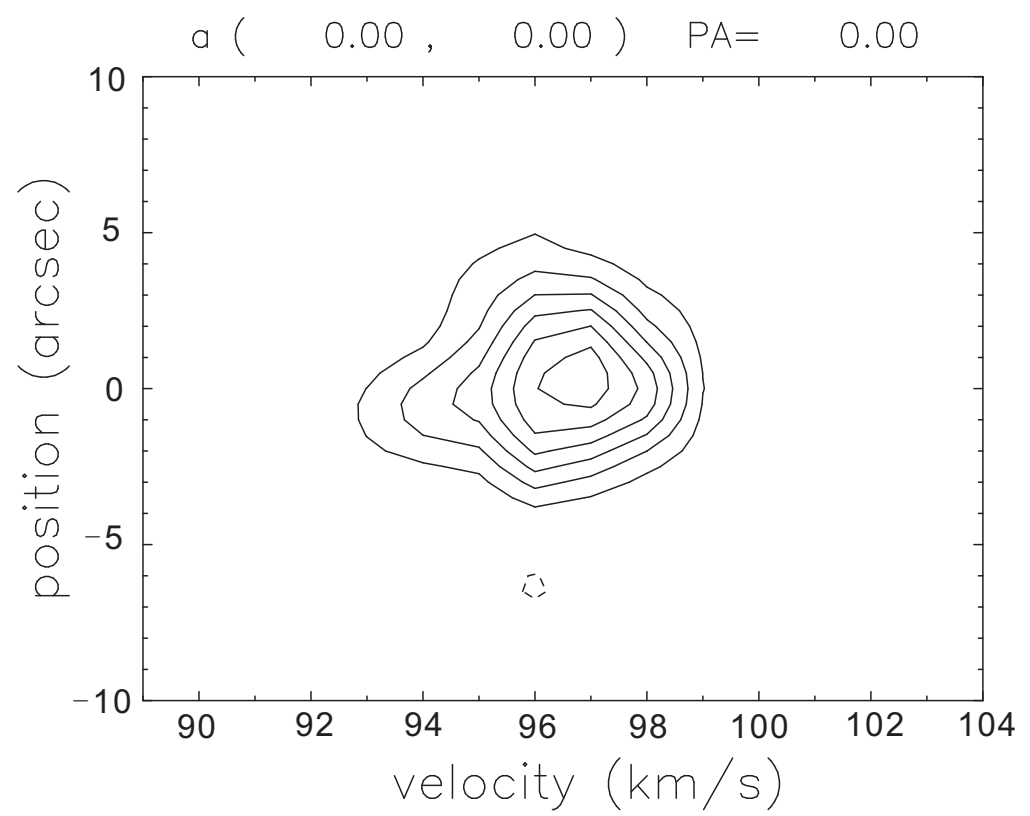

Fig. 3.- Position-velocity diagrams of $\mathrm{CH}_{3} \mathrm{OH}$ along a P.A. of $0^{\circ}$. The contour levels are from $15 \%$ to $90 \%$ in steps of $15 \%$ of the peak intensity in both panels. The intensity at the peak is $3.73 \mathrm{Jy}^{\text {beam }^{-1}}$. 

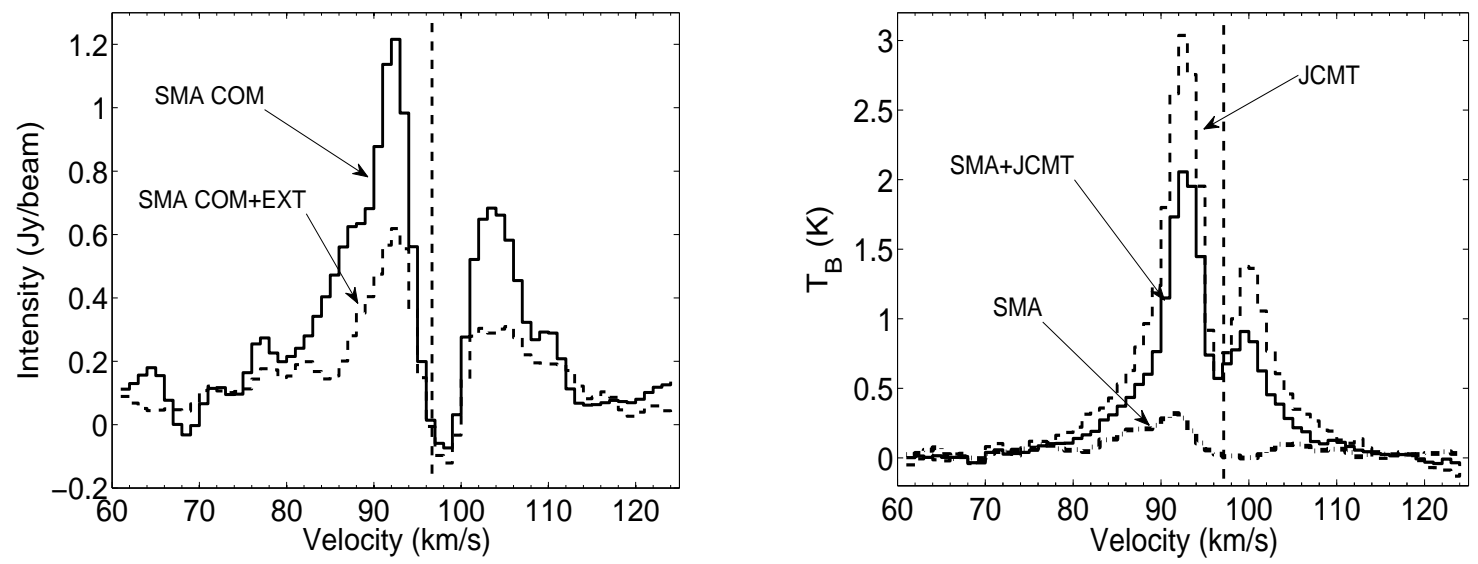

Fig. 4.- The HCN (3-2) spectra. Left: the solid black line exhibits the spectrum constructed from SMA compact array and the dashed line shows the spectrum obtained from combining the compact and extended data together. Both of the two spectra are integrated over a region of $5^{\prime \prime} \times 5^{\prime \prime}$. Right: the solid black line shows the spectrum constructed from the combined SMA and JCMT data, which is convolved with the JCMT beam (18.3"); the dashed line shows the spectrum from JCMT only; the dash-dotted gray line shows the spectrum obtained with the SMA compact array and convolved with the JCMT beam. The vertical dashed lines in both panels mark the position of the systematic velocity $\left(96.7 \mathrm{~km} \mathrm{~s}^{-1}\right)$. 


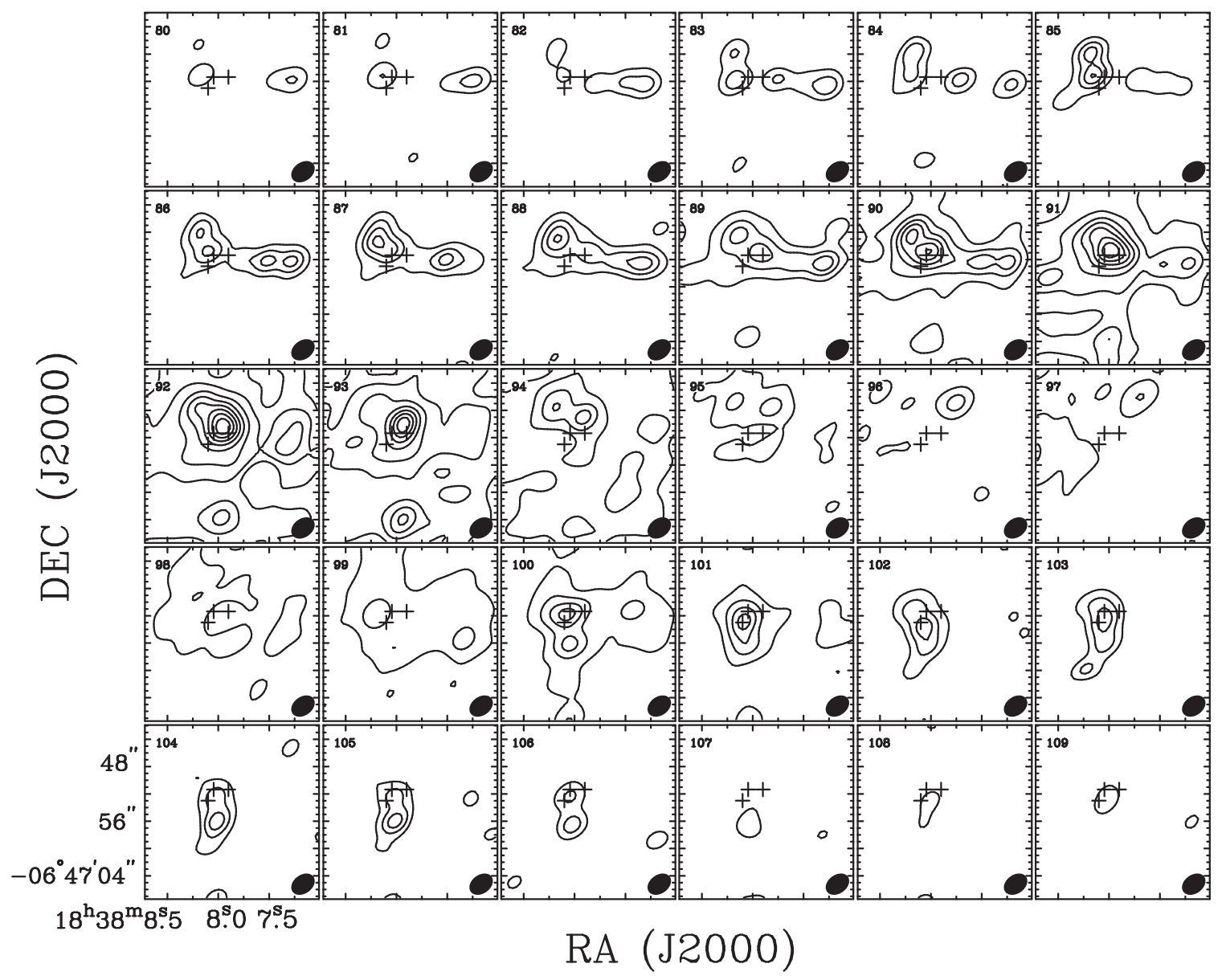

Fig. 5.- Combined JCMT and SMA HCN (3-2) channel maps from $80 \mathrm{~km} \mathrm{~s}^{-1}$ to 109 $\mathrm{km} \mathrm{s}^{-1}$, which is convolved with the beam of the SMA compact configuration $\left(3.76^{\prime \prime} \times 2.72^{\prime \prime}\right.$, $\left.\mathrm{PA}=-54^{\circ}\right)$. The contours are in steps of $0.5 \mathrm{Jy} \mathrm{beam}^{-1}(3 \sigma)$ from $0.5 \mathrm{Jy} \mathrm{beam}^{-1}(3 \sigma)$. The velocity of each channel is plotted at the upper-left of each panel, and the beam size at the lower-right. The positions of MM1, MM2 and MM3 are marked with crosses. 

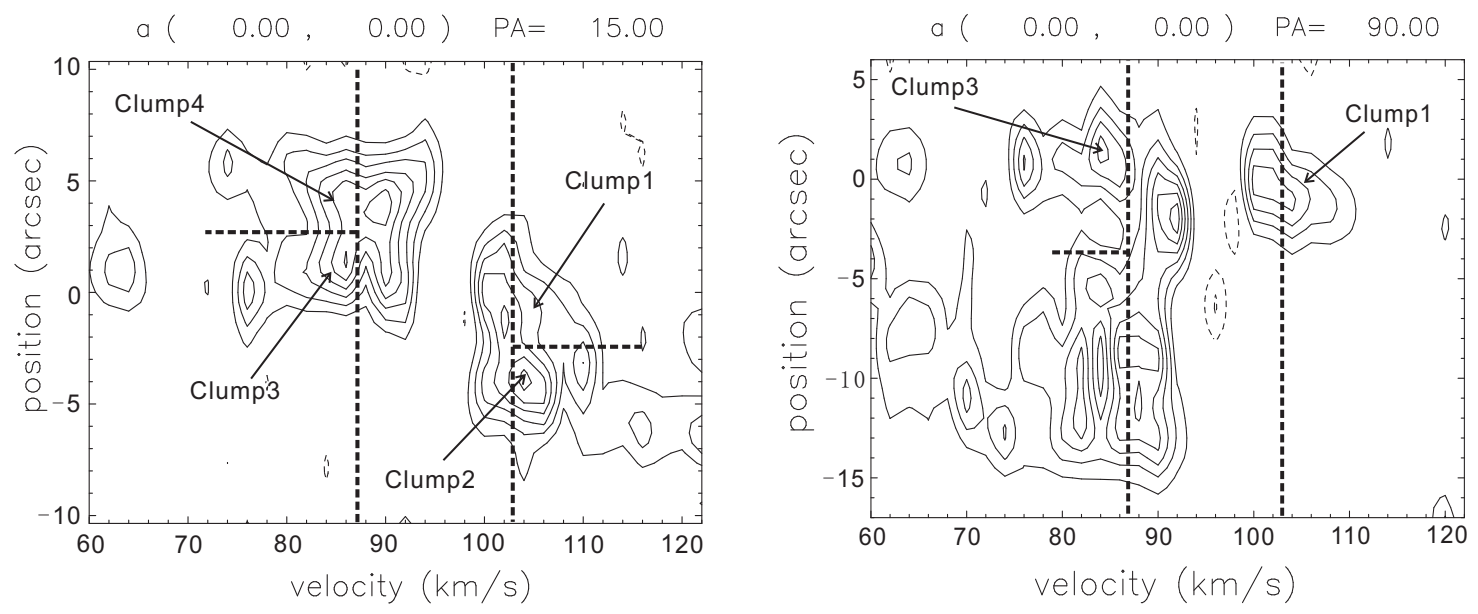

Fig. 6.- Position-velocity diagrams of HCN (3-2) outflow observed by the SMA along a P.A. of $15^{\circ}$ (left panel) and $90^{\circ}$ (right panel). The image is smoothed to $2 \mathrm{~km} \mathrm{~s}^{-1}$ velocity resolution. The contour levels are from $15 \%$ to $90 \%$ in steps of $15 \%$ of the peak intensity in both panels. The peak is $1.84 \mathrm{Jy} \mathrm{beam}^{-1}$ in the left panel and $1.92 \mathrm{Jy} \mathrm{beam}^{-1}$ in the right panel. The four clumps are labeled by solid lines with arrows. The clumps are distinguish by the thick dashed lines. 


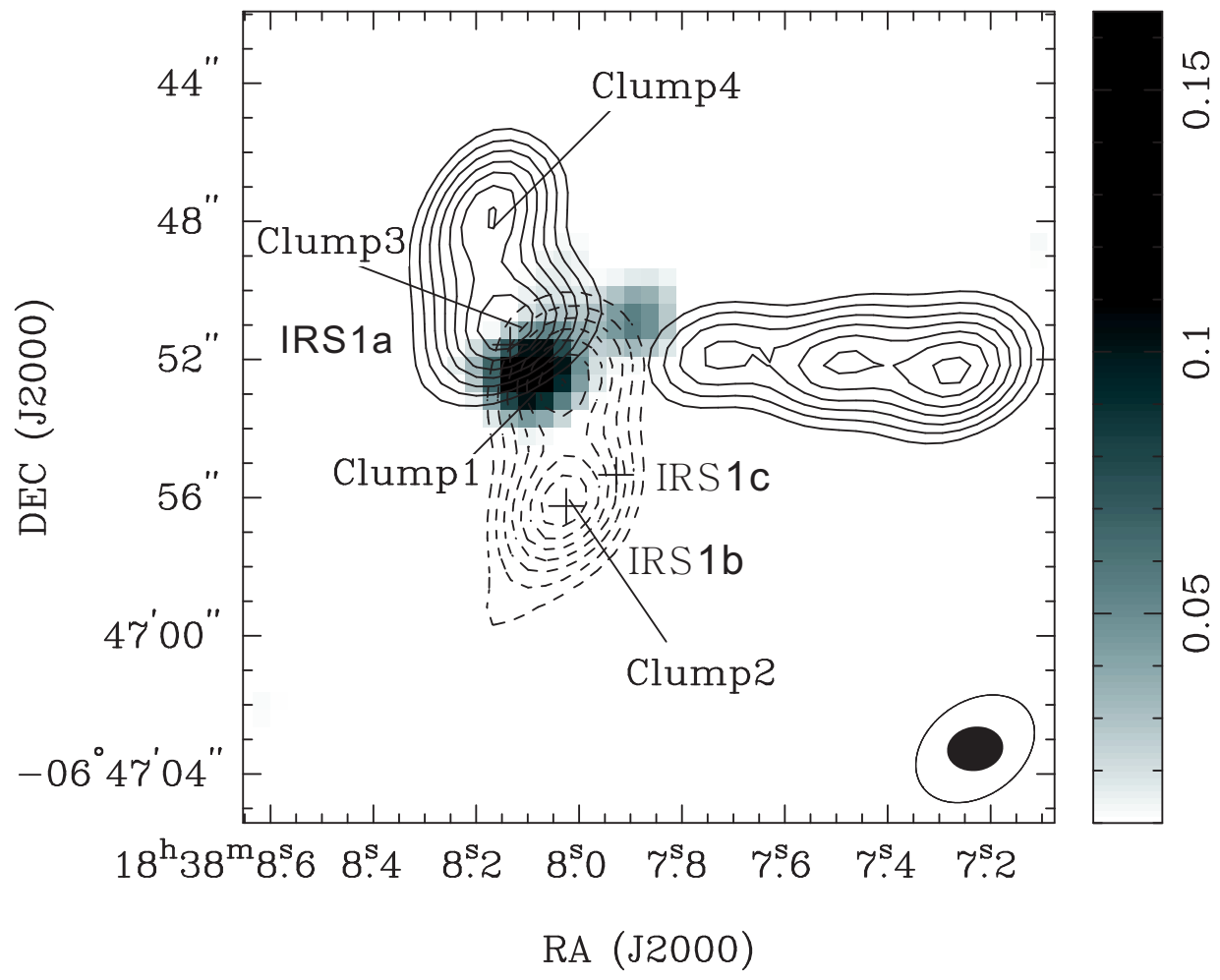

Fig. 7.- The high-velocity HCN (3-2) intensity contours overlaid on the $1.3 \mathrm{~mm}$ continuum image, integrated from 80 to $87 \mathrm{~km} \mathrm{~s}^{-1}$ for the blueshifted lobes (solid contours) and from 103 to $109 \mathrm{~km} \mathrm{~s}^{-1}$ for the redshifted lobe (dashed contours), with contours from $30 \%$ in steps of $10 \%$ of the peak emission. The peak is $8.55 \mathrm{Jy} \mathrm{beam}^{-1} \cdot \mathrm{km} \mathrm{s}^{-1}$ for the blueshifted lobes and $7.16 \mathrm{Jy}$ beam ${ }^{-1} \cdot \mathrm{km} \mathrm{s}^{-1}$ for the redshifted lobe. The empty and solid ellipses in the lower-right corner represent the synthesized beams of HCN (3-2) emission and $1.3 \mathrm{~mm}$ continuum emission combined from both configurations, respectively. 